\title{
Deposition of metallic clusters on a metallic surface at zero initial kinetic energy: Evidence for implantation and site exchanges
}

\author{
B. Nacer \\ Département de Physique, Faculté des Sciences et Techniques, Université Cady Ayyad, Guéliz Marrakech, Morocco \\ C. Massobrio \\ Institut de Physique et de Chimie des Matériaux de Strasbourg, 23, rue de Loess, F-67037 Strasbourg, France \\ C. Félix \\ Bell Laboratories, Lucent Technologies, Murray Hill, New Jersey 07974-0636 \\ and Institut de Physique Expérimentale, Ecole Polytechnique Fédérale de Lausanne, \\ PHB-Ecublens, CH-1015 Lausanne, Switzerland
}

(Received 27 January 1997)

\begin{abstract}
We have investigated the deposition at zero impact kinetic energy of the Ag atom and clusters $\left(\mathrm{Ag}_{7}, \mathrm{Ag}_{19}\right)$ on the (100) and (111) surfaces of Pd by molecular-dynamics simulations performed within the embeddedatom-method scheme. Our results elucidate the role played by the adsorption energy in determining the final morphology of the cluster/substrate system when ideal nondestructive deposition conditions are implemented. While implantation of the atom is not observed, we find a finite probability of site Ag-Pd exchanges in the case of clusters. Deposition-assisted mixing occurring at the topmost surface layer appears to be correlated to the size of the cluster and the orientation of the substrate, being higher for $\mathrm{Ag}_{7} / \mathrm{Pd}(100)$ and lower for $\mathrm{Ag}_{19} / \mathrm{Pd}(111)$. Total-energy calculations, combined with an analysis of the atomic motion, indicate that the structural transformation accompanying the deposition of the cluster provides the needed activation energy to induce the observed Ag-Pd atomic exchanges. [S0163-1829(97)00640-1]
\end{abstract}

\section{INTRODUCTION}

Experiments on cluster size selection and deposition on solid surfaces at low impact energies ("soft landing") are appealing because of their innovative character with respect to the fabrication and investigation of nanoscale materials. ${ }^{1-4}$ These collisions attract consideration from both cluster and surface physics communities, since they render possible the addition of a new parameter (i.e., the size of the deposited units) to the investigation of growth and dynamics on surfaces.

Recently, experiments of size selection and cluster deposition have been performed on the system $\mathrm{Ag}_{n}^{+} / \mathrm{Pd}(100)$ using thermal-energy atom scattering. ${ }^{4-6}$ In combination with molecular-dynamics simulations, these investigations have shown that by increasing the cluster size fragmentation decreases, while the deposited structures become more compact and structurally ordered. However, implantation of Ag atoms cannot be avoided ${ }^{6}$ even at a collision energy per atom as low as $E_{c} \sim 1 \mathrm{eV}$. In view of these results, the question arises as to the possibility of achieving real soft-landing conditions, featuring a defect-free interface when $E_{c}$ goes to zero. In principle, the $\mathrm{Ag} / \mathrm{Pd}(100)$ system is well suited to meeting this requirement. In fact, the two metals do not mix if $\mathrm{Ag}$ is thermally evaporated on $\operatorname{Pd}(100)$ at temperatures lower than $T=550 \mathrm{~K} .^{7}$ Furthermore, from the standpoint of energetics, embedded-atom-method (EAM) calculations have shown that $\mathrm{Ag}$ is more stable on $\operatorname{Pd}(100)$ than embedded in it. ${ }^{8}$

It is worth mentioning that in the case of atomic deposition extreme soft-landing conditions can be straightforwardly obtained through atomic evaporation from a thermal source. The kinetic energy associated with this process is essentially negligible compared with the adsorption energy $E_{\text {ad }}$ released upon condensation of the atom on the substrate. As shown in Ref. 9, nonthermal ("ballistic', or transient) exchanges, driven by $E_{\mathrm{ad}}$, can occur between impinging and substrate atoms of the first layer at temperatures much lower than those needed for thermal migration. However, the transfer of the condensation energy into motion along the surface is inefficient and does not allow us to assess unambiguously whether or not extended transient mobility on the surface exists. Molecular-dynamics simulations have attributed the lack of transient mobility to the rapid transfer of adsorption energy toward the substrate. ${ }^{10,11}$ In particular, Raeker, Perkins, and Yang ${ }^{10}$ have shown that, depending on the diffusion parameters, the local heating brought about by the deposition can induce transient exchanges that may have a longrange nature.

In this paper we establish a link between cluster deposition at zero impact energy and the resulting dynamical processes driven by the adsorption energy of the cluster to the substrate. To this purpose we carried out moleculardynamics simulations of the deposition of $\mathrm{Ag}_{1}, \mathrm{Ag}_{7}$, and $\mathrm{Ag}_{19}$ on $\mathrm{Pd}(100)$ and $\mathrm{Pd}(111)$ at zero impact energy by using EAM interatomic potentials. ${ }^{8}$ Our results show that cluster deposition at very low energy can induce surface alloying via the occurrence of nonthermal site exchanges between cluster and substrate atoms. This phenomenon is triggered by the structural transformation from a three-dimensional to a more planar adsorbed structure undergone by the cluster during deposition. The related gain in adsorption energy provides 
the activation energy necessary to promote atomic exchanges. We show that the amplitude of this effect depends on the size of the cluster and on the orientation of the substrate, this latter determining the exchange barriers for adatom migration.

This paper is organized as follows. Section II describes the model used and its practical implementation in the context of atom and cluster deposition. The first part of Sec. III is devoted to the deposition of $\mathrm{Ag}_{1}$ while the second one deals with the systems $\mathrm{Ag}_{7}$ and $\mathrm{Ag}_{19}$ on both $\mathrm{Pd}(100)$ and $\mathrm{Pd}(111)$. A detailed analysis of the dynamics of the impact process is carried out in the specific case of $\mathrm{Ag}_{7} / \mathrm{Pd}(100)$. Conclusive remarks are collected in Sec. IV.

\section{MODEL AND CALCULATIONS}

Our simulations are based on the EAM potentials of Ref. 8 for $\mathrm{Ag}$ and $\mathrm{Pd}$, with cutoff radii $R_{\text {cut }}$ equal to $5.25 \AA$. The $\mathrm{Ag}-\mathrm{Ag}$ repulsive part is modified by the addition of an interaction $E_{R}=A e^{-\left(r-r_{d}\right) \lambda}$ and then refitted to the cohesive energy and equilibrium distance of $\mathrm{Ag}_{7}$ in its ground state $\left(D_{5 h}\right.$ symmetry) determined via first-principles calculations. ${ }^{13}$ Purely two-body repulsive interactions are achieved by switching off the attractive part of the EAM potentials for a value of the electronic density corresponding, in the bulk, to a distance $80 \%$ smaller than the bulk interatomic nearestneighbor distance. The description of isolated metallic clusters via $n$-body interatomic potentials should be considered appropriate only within the framework of cluster-substrate collisions, being that the EAM scheme is intrinsically unsuitable to model small clusters in the gas phase.

In the case of $\operatorname{Pd}(100)$, the simulation slab consists of seven layers, modeling the (100) surface of Pd and submitted to periodic boundary conditions along the [001] and [010] directions. Each layer contains 200 Pd atoms. Similarly, for $\mathrm{Pd}(111)$, the slab is modeled by six layers with $200 \mathrm{Pd}$ atoms each, oriented along the [110] and [1 12] directions. These system sizes are comparable to those employed in the literature to simulate thermal depositions of atoms, ${ }^{10,11,14}$ and not too large $(N \sim 100)$ clusters. ${ }^{15}$ We deposited heptamers having the $D_{5 h}$ (a pentagonal bipyramid) and the $C_{3 v}$ symmetry, the latter corresponding to four coplanar atoms facing one atom on one side and two atoms on the opposite side. These structures resulted from an extended search of equilibrium structures carried out by running molecular-dynamics cycles at temperatures in between $T=500$ and $800 \mathrm{~K}$, followed by quench at $T=0 \mathrm{~K}$. The same approach for the $\mathrm{Ag}_{19}$ cluster leads to several local minima, from which we selected a highly distorted six-capped icosahedron as initial configuration. We found it instructive to consider also planar $\mathrm{Ag}_{7}$ structures arranged in the most compact forms compatible with the (100) and (111) substrates. These planar clusters, unstable in the free space, do not suffer from important distortions when approaching the slabs from a distance $R$ $<R_{\text {cut }}$.

In the simulations of $\mathrm{Ag}_{1} / \mathrm{Pd}(100)$ and $\mathrm{Ag}_{1} / \mathrm{Pd}(111)$ the initial distance between atom and substrate corresponds to a value slightly smaller than the interaction cutoff. The Ag atom approaches the surface along the [100] (or [111]) direction at zero initial kinetic energy, with the system initially prepared at $T=0 \mathrm{~K}$. In the case of $\mathrm{Ag}_{7}$ and $\mathrm{Ag}_{19}$, only one atom is taken to feel the attraction of the surface at $t=0$ (atoms have equal heights above the substrate when the heptamer is planar). For each cluster-substrate combination 100 depositions are produced for statistical purposes, by varying the atom or cluster location with respect to the (100) and (111) planes. The evolution of the system takes place in the microcanonical ensemble, with trajectories lasting up to 20 ps. This length is sufficient to reach thermodynamical equilibrium, as controlled by monitoring the evolution of cluster and substrate temperatures following the impact. Our results are unaffected by the consideration of a different, low ( $T$ $=120 \mathrm{~K}$ ) initial substrate temperature or by the inclusion of a small initial kinetic energy of the impinging atom or cluster (0.1 eV/atom).

Static calculations have also been performed to evaluate the energetics of adatoms and adsorbed clusters and the diffusion barriers at $T=0 \mathrm{~K}$. Relaxation to this temperature has been achieved by smooth quenching via the application of the Nosé thermostat. ${ }^{16}$ Calculation of diffusion barriers is carried out by constraining the $\mathrm{Ag}$ atom to migrate (or the Ag-Pd pair in the case of exchange) at fixed positions along a given diffusion path. The diffusion path is along the [110] (hopping) and [001] (exchange)directions in the case of the (100) substrate and along the [1-10] (hopping) and [1 $\overline{2} 1]$ (exchange) directions in the case of the (111) substrate.

In what follows we shall refer to $E_{N}^{2 \mathrm{D}}$, the total energy relative to the most stable $\mathrm{Ag}_{N} / \mathrm{Pd}$ configuration at $T$ $=0 \mathrm{~K}, E_{N}^{\text {ini }}$, the sum of the total energies of the slab and cluster noninteracting and $E_{N}^{\mathrm{fin}}$, the total energy of the system relaxed at $T=0 \mathrm{~K}$ after completion of the deposition. The adsorption energy for the whole deposition process is therefore $E_{N}^{\mathrm{fin}}-E_{N}^{\mathrm{ini}}$. Since three-dimensional (3D) clusters are unstable on both (100) and (111) surfaces we need a definition of a reference total energy for the 3D cluster-substrate system $E_{N}^{3 \mathrm{D}}$. To this purpose we take the cluster in its initial shape and we position it on $\operatorname{Pd}(100)$ [or $\operatorname{Pd}(111)]$. Its lower atom is placed at about a bulk Pd-Pd interplanar distance above the substrate. To avoid structural modifications in the cluster, only the substrate atoms are left free to relax at $T$ $=0 \mathrm{~K}$. Although not univocal, this definition allows to relate the observed occurrences of site exchanges to the relevant quantities (exchange barriers and adsorption energies) of the system under consideration.

\section{RESULTS}

\section{A. Atom depositions}

We have investigated atomic deposition for the systems $\mathrm{Ag}_{1} / \mathrm{Pd}(100)$ and $\mathrm{Ag}_{1} / \mathrm{Pd}(111)$ at zero impact energy. In agreement with the results of Ref. 10, our simulations confirm that the deposition of $\mathrm{Ag}$ on $\operatorname{Pd}(100)$ [and $\mathrm{Pd}(111)$ ] takes place with no defect production and, in particular, no site exchange is observed. Following Kellogg, ${ }^{9}$ we analyze these results considering the exchange barrier $E_{\text {exc,(100) }}$ $=0.75 \mathrm{eV}$ and the adsorption energy $E_{\mathrm{ad} 1}=-2.97 \mathrm{eV}$ for $\mathrm{Ag}_{1}$ on $\mathrm{Pd}(100)$. These energies yield a probability of $\mathrm{Ag}-\mathrm{Pd}$ exchanges as high of $77 \%$ when inserted in a Boltzmann distribution of exchange attempts, i.e., $e^{\left(E_{\mathrm{exc}}\right) / E_{\mathrm{ad} 1}}$. Such unrealistically high rate follows from the total neglect of the kinetic aspects of the deposition and, in particular, of the rate 
TABLE I. Calculated quantities describing $\mathrm{Ag}_{7}$ and $\mathrm{Ag}_{19}$ cluster depositions on $\operatorname{Pd}(100)$ and $\operatorname{Pd}(111)$. $P_{\text {exc }}^{1}$ is the number of depositions (over a total of 100) giving Ag-Pd site exchanges, within parentheses the number of exchanges involving only a single Ag-Pd pair. $\Delta E^{3 \mathrm{D}-2 \mathrm{D}}$ is the difference $E_{N}^{3 \mathrm{D}}-E_{N}^{2 \mathrm{D}}$ (see text). $\Delta E^{3 \mathrm{D}-\text { fin }}$ and $\Delta E^{\text {ini-3D }}$ are equal to $E_{N}^{3 \mathrm{D}}-E_{N}^{\text {fin }}$ (with the value per atom in parentheses), and $E_{N}^{\text {ini }}-E_{N}^{3 \mathrm{D}}$, respectively. For $\mathrm{Ag}_{7}$ we report the data relative to the $C_{3 v}$ structure only, (a similar number of exchanges has been found in the case of the $D_{5 h}$ ) and in addition the value of $P_{\mathrm{exc}}^{1}$ for the planar structure. $E_{\mathrm{exc}(100)}$ and $E_{\text {exc(111) }}$ are the atomic exchange barriers for $\mathrm{Ag}_{1}$ on $\mathrm{Pd}(100)$ or $\mathrm{Pd}(111) . \quad P_{\text {exc }}^{2}$ is probability of exchange obtained by employing the Boltzmann factor on the basis of the calculated differences of total energies between the $3 \mathrm{D}$ and the final configurations (see text).

\begin{tabular}{|c|c|c|c|c|c|}
\hline & $\begin{array}{c}-\Delta E^{3 \mathrm{D}-2 \mathrm{D}} \\
(\mathrm{eV})\end{array}$ & $\begin{array}{c}-\Delta E^{\text {ini-3D }} \\
(\mathrm{eV})\end{array}$ & $\begin{array}{c}-\Delta E^{3 \mathrm{D}-f i n} \\
(\mathrm{eV})\end{array}$ & $P_{\mathrm{exc}}^{1}$ & $P_{\text {exc }}^{2}$ \\
\hline $\mathrm{Ag}_{7} / \mathrm{Pd}(100)$ & 7.64 & 2.42 & $5.68(0.81)$ & $79(68)$ & 39 \\
\hline $\mathrm{Ag}_{7} / \mathrm{Pd}(111)$ & 5.97 & 1.94 & $4.56(0.65)$ & $37(37)$ & 28 \\
\hline $\mathrm{Ag}_{19} / \mathrm{Pd}(100)$ & 17.50 & 4.49 & $8.08(0.42)$ & $49(39)$ & 17 \\
\hline $\mathrm{Ag}_{19} / \mathrm{Pd}(111)$ & 13.39 & $\begin{array}{c}4.09 \\
-\Delta E^{\mathrm{ini}-\mathrm{fin}} \\
(\mathrm{eV})\end{array}$ & $7.08(0.37)$ & $10(10)$ & 10 \\
\hline $\mathrm{Ag}_{7}^{\text {planar }} / \mathrm{Pd}(100)$ & & 14.55 & & 0 & \\
\hline $\mathrm{Ag}_{7}^{\text {planar }} / \mathrm{Pd}(111)$ & & 13.11 & & 0 & \\
\hline $\begin{array}{l}E_{\text {exc }}[\mathrm{Ag} / \mathrm{Pd}(100)]=0.75 \mathrm{eV} \\
E_{\text {exc }}[\mathrm{Ag} / \mathrm{Pd}(111)]=0.82 \mathrm{eV}\end{array}$ & & & & & \\
\hline
\end{tabular}

of energy transfer from the atom to the substrate. Indeed, the kinetic energy of the incoming atom is rapidly transferred to the substrate, with accomodation in a stable adsorption site accomplished in a time no longer than 1 ps. In agreement with previous investigations carried out in Refs. 10 and 11 we conclude that this loss of energy prevents transient exchanges from taking place, since the associated barrier is much too high for this process to occur within the time scale typical of the deposition process. ${ }^{17}$

\section{B. Cluster depositions}

The total-energy differences between 2D and 3D Ag structures in $\operatorname{Pd}(100)$ and $\operatorname{Pd}(111)$ are reported in the first column of Table I. These values clearly indicate that on both substrates energetics favors planar structures maximizing atomic coordination. Therefore, as a result of deposition, a transformation toward a more planar structure is expected, consistently with previous MD results regarding energetic collisions. $^{6,12}$

Our simulations indicate that all deposited heptamers are planar after completion of the impact process. Disruption of the original 3D structure is observable also in the case of $\mathrm{Ag}_{19}$, which arranges mostly in two-layer structures. The lowest-energy configurations and representative examples of geometries obtained after deposition are given in Figs. 1 and 2. The final configurations are not only characterized by shapes different from the most stable ones. In addition, a relevant number of Ag-Pd site exchanges takes place. These two reasons account for the increased value of $\Delta E^{3 \mathrm{D}-\mathrm{fin}}$ with respect to $\Delta E^{3 \mathrm{D}-2 \mathrm{D}}$. From Table I we note that the number of depositions $P_{\text {exc }}$ leading to implantation of at least one $\mathrm{Ag}$ atom ranges from 79 (over a total of 100) in the case of $\mathrm{Ag}_{7} / \mathrm{Pd}(100)$ to 10 in the case of $\mathrm{Ag}_{19} / \mathrm{Pd}(111)$, while implantation of two Ag atoms is much less frequent and does not occur in the case of (111) substrates. We do not find any
$\mathrm{Ag}$ atom in an interstitial position, as for each $\mathrm{Ag}$ implanted a Pd atom is promoted on the substrate. The values of $P_{\text {exc }}$ correlate well to the values of $-\Delta E^{3 \mathrm{D}-\mathrm{fin}} / N$. This quantity turns out to be smaller for $\mathrm{Ag}_{19}$ than $\mathrm{Ag}_{7}$ and takes the largest value for $\mathrm{Ag}_{7} / \mathrm{Pd}(100)$. The degree of implantations appears to be dependent on the size of the cluster and the orientation of the substrate. We found $P_{\text {exc }}$ to be larger on
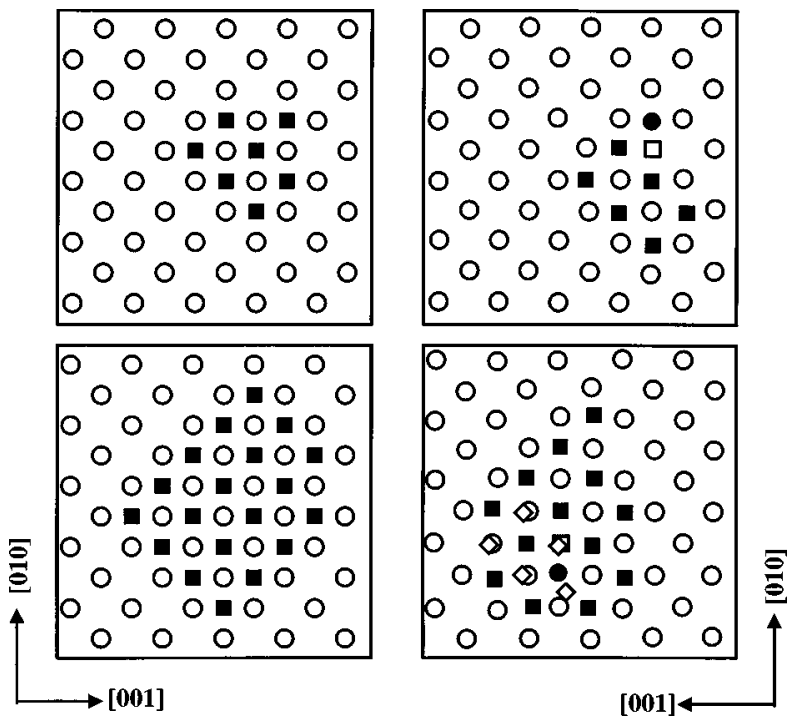

FIG. 1. Left part: most stable geometries calculated at $T=0 \mathrm{~K}$ for $\mathrm{Ag}_{7}$ on $\mathrm{Pd}(100)$ (top) and $\mathrm{Ag}_{19}$ on $\mathrm{Pd}(100)$ (bottom). Filled squares, Ag atoms; open circles, Pd atoms in the uppermost layer. Right part: one example of final arrangement obtained at the end of the deposition for $\mathrm{Ag}_{7}$ on $\mathrm{Pd}(100)$ (top) and $\mathrm{Ag}_{19}$ on $\mathrm{Pd}(100)$ (bottom). Circles are Pd atoms, squares and rhombuses are Ag atoms. Filled circles stand for atoms in the first adlayer, white squares for $\mathrm{Ag}$ implanted atoms and white rhombuses for $\mathrm{Ag}$ atoms in the second adlayer. 

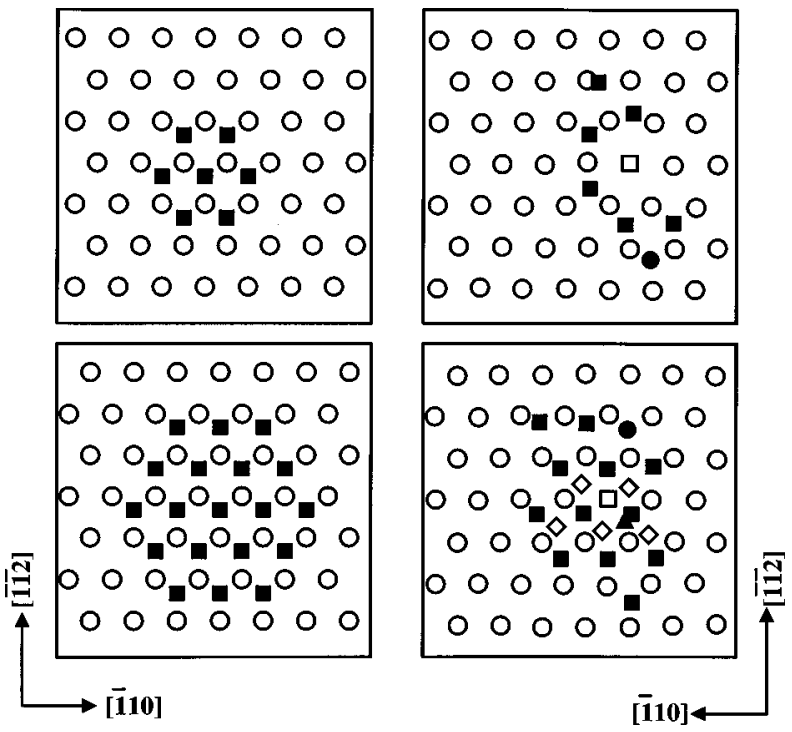

FIG. 2. Left part: most stable geometries calculated at $T=0 \mathrm{~K}$ for $\mathrm{Ag}_{7}$ on $\mathrm{Pd}(111)$ (top) and $\mathrm{Ag}_{19}$ on $\mathrm{Pd}(111)$ (bottom). Right part: one example of the final arrangment obtained at the end of the deposition for $\mathrm{Ag}_{7}$ on $\mathrm{Pd}(111)$ (top) and $\mathrm{Ag}_{19}$ on $\mathrm{Pd}(111)$ (bottom). The meaning of the symbols is the same as in Fig. 1, with the addition of a filled triangle, corresponding to an Ag atom lying at the third-layer level.

$\operatorname{Pd}(100)$ than on $\operatorname{Pd}(111)$, where the migration barrier for Ag-Pd exchanges is higher. Remarkably, no implantation occurs when a planar $\mathrm{Ag}_{7}$ is deposited.

These results suggest two considerations. First, due to the kinetics of the process, the simple transfer of kinetic energy from the cluster to the substrate is not sufficient to trigger site exchanges. In fact, the planar structure releases as much as $14.55 \mathrm{eV}$ toward the $\mathrm{Pd}(100)$ substrate without inducing any surface defect. On the other hand, when a structural transition accompanies the deposition, we observe a much higher probability of implantation. To understand why this happens we consider the variation in time of the quantities displayed in Fig. 3 for the case of $\mathrm{Ag}_{7} / \mathrm{Pd}(100), \mathrm{Ag}_{7}$ being initially in the $C_{3 v}$ symmetry. We note that $T_{\mathrm{cm}}$, the kinetic energy of the cluster center of mass, increases abruptly over a very short time interval, attains its maximum, and then comes down to its first minimum, reached at $t=1.7 \mathrm{ps}$. The transfer of energy to the substrate begins already for $t>1 \mathrm{ps}$ and takes place, at least for $t<1.7 \mathrm{ps,} \mathrm{without} \mathrm{any} \mathrm{important}$ structural changes in the cluster, which keeps its shape basically unchanged. In the inset of Fig. 3, the average distance of all particles from their center of mass $R_{\mathrm{cm}}$ shows only a limited increase within the time interval $1-1.7 \mathrm{ps}$ and, over the same interval, the cluster internal temperature $T_{\text {int }}$ goes up to $T \sim 400 \mathrm{~K}$.

Visual inspection of the snapshots shown in Fig. 4 provides further evidence on the almost exclusively translational character of the heptamer motion when approaching the substrate, for $t<1.8 \mathrm{ps}$. However, for longer times, we observe the structural transformation of the cluster. Its translational motion diminishes notably resulting in the decay of $T_{\mathrm{cm}}$ between $t=2$ and 4 ps (see Fig. 3) while $T_{\text {int }}$ exhibits a maximum at $t=2.1 \mathrm{ps}$ and a subsequent decrease, leading to the recovery of thermal equilibrium conditions. The radical transformation of the cluster structure is further highlighted
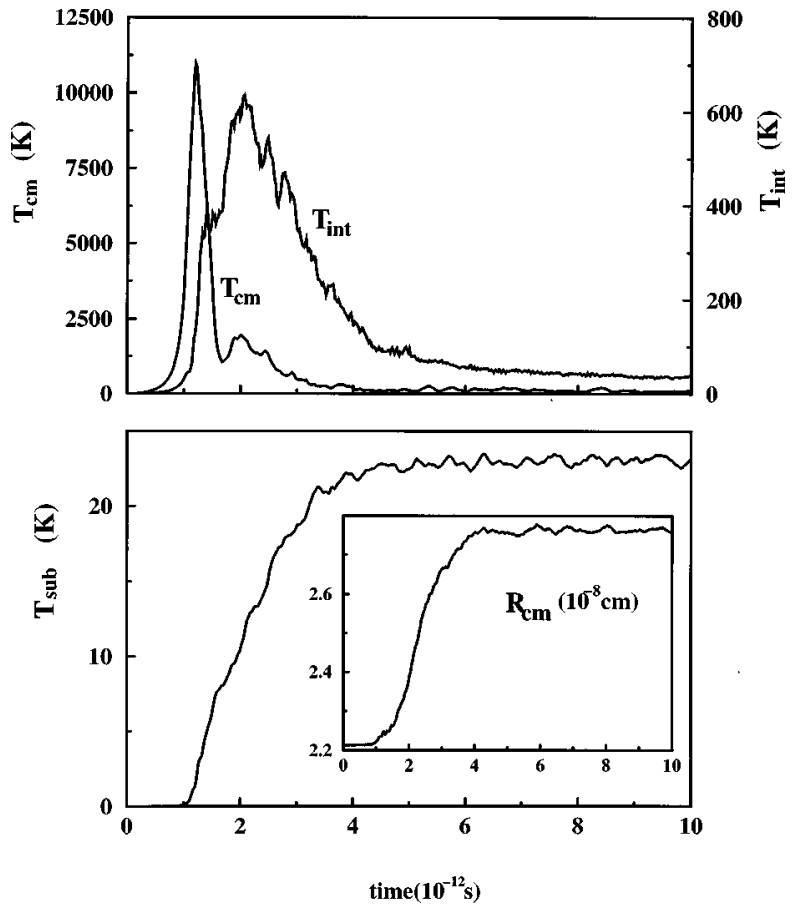

FIG. 3. Upper part: temporal evolution of the kinetic energy of the center of mass $T_{\mathrm{cm}}$ (expressed in units of temperature) and of the internal temperature of the cluster $T_{\text {int }}$, calculated in the center of mass reference frame in the case of $\mathrm{Ag}_{7} / \mathrm{Pd}(100)$. Lower part: temporal evolution of the substrate temperature $T_{\text {sub }}$ in the case of $\mathrm{Ag}_{7} / \mathrm{Pd}(100)$. Inset in the lower part: temporal evolution of $R_{\mathrm{cm}}$ (the average atomic distance from the cluster center of mass) in the case of $\mathrm{Ag}_{7} / \mathrm{Pd}(100)$. In the inset the scale of time is omitted for clarity. The data are averages over 100 depositions.

by the behavior of $R_{\mathrm{cm}}$, which goes through a rapid increase for $t>2 \mathrm{ps}$ and stabilizes at $t \sim 4 \mathrm{ps}$ (Fig. 3 , inset). In the sequences presented in Fig. 4, the cluster first seeks to arrange in two layers $(1.7<t<2.4 \mathrm{ps})$ and then a $\mathrm{Ag}$-Pd site exchange takes place along the [001] direction via a concerted motion consisting of a direct $\mathrm{Ag}$-Pd replacement that lasts $\sim 0.5$ ps. After accomplishment of the replacement sequence, no further transient mobility is observed. All exchanges involve $\mathrm{Pd}$ atoms in the uppermost layer only. A non-negligible fraction of these exchanges have indirect nature. $^{18}$

These observations lend support to a description of the deposition process based on two distinct steps. The first is the approach of the cluster to the substrate, driven by the adsorption energy $\Delta E^{\text {ini-3D }}$. This part is characterized by negligible structural transformation and limited thermal motion of the cluster. In the second step the cluster (and to a lesser extent, the substrate) rearranges to find the most stable geometry. Now the relevant energy is $\Delta E^{3 \mathrm{D} \text {-fin }}$. Translational motion of the cluster is progressively suppressed, while thermal motion is enhanced. In about 2 ps the final configuration is attained and the adsorption energy is redistributed among all degrees of freedom. We found that the energy associated with the structural transformation can activate implantation of atoms on the substrate via exchange processes. The amount of exchanges appears to be well correlated with the value of $\Delta E^{3 \mathrm{D}-\text { fin }} / N$. By using this quantity and the atomic exchange barriers one can estimate a lower bound for the probability of implantation $P_{\mathrm{exc}}^{2}=e^{-\left(E_{\mathrm{exc}}\right) / E_{\mathrm{ad} 1}}$, where $E_{\mathrm{ad} 1}$ 

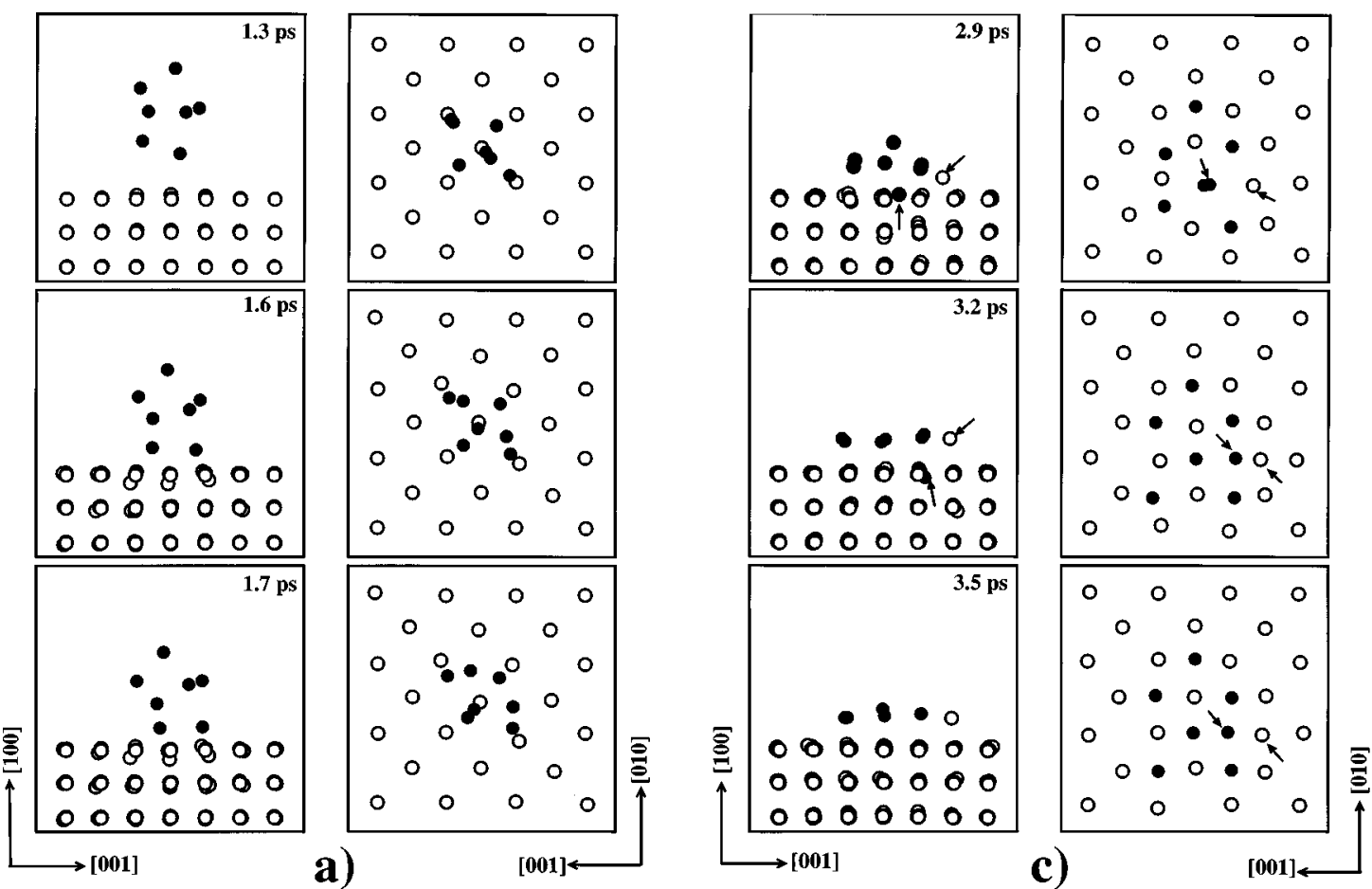

a)
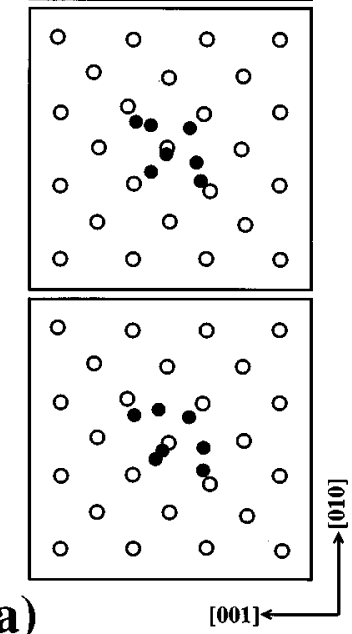

c)

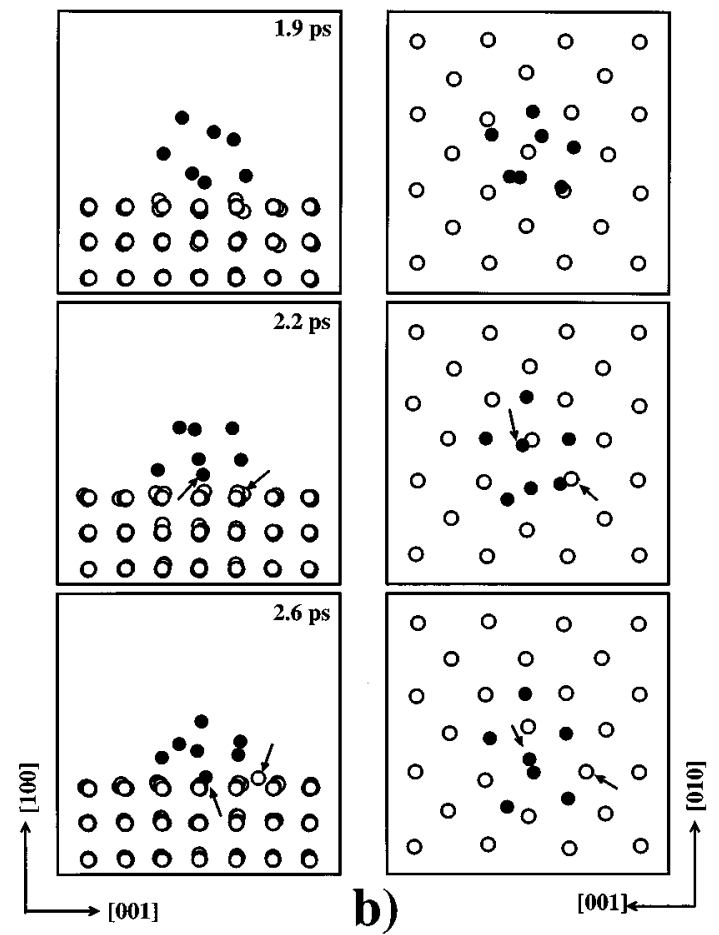

FIG. 4. Sequence of snapshots showing the deposition of $\mathrm{Ag}_{7}$ on $\mathrm{Pd}(100)$ in one particular, representative case. (a), $t=1.3,1.6$, and 1.7 ps; (b), $t=1.9,2.2$, and $2.6 \mathrm{ps}$; (c), $t=2.9,3.2$, and $3.5 \mathrm{ps}$. The indicated times are taken with respect to the beginning of the deposition. Filled circles represent $\mathrm{Ag}$ atoms and empty circles represent Pd atoms. For each panel, in the left part three layers of Pd atoms are shown, and the coordinates are projected onto the (100) plane. In the right part the coordinates are projected onto the surface (001) plane and only the uppermost original $\mathrm{Pd}$ atoms are shown. The arrows indicate the atoms undergoing a site exchange. $\mathrm{Ag}_{7}$ is initially in the $C_{3 v}$ symmetry.

this time is equal to $-\Delta E^{3 \mathrm{D}-\mathrm{fin}} / N$. As shown in Table I the trend exhibited by $P_{\mathrm{exc}}^{1}$ is reproduced by $P_{\mathrm{exc}}^{2}$, although the choice for the value of $E_{\text {ad } 1}$ gives better results in the case of $\mathrm{Ag}_{N} / \mathrm{Pd}(111)$. Overall larger cluster sizes and more compact substrates concur to reduce surface alloying induced by cluster deposition. This is due to the following two factors: (a) with increasing cluster size the energy per atom available to overcome exchange barriers becomes smaller and (b) more compact substrates correspond to higher exchange barriers. In view of these arguments, it is plausible to conjecture that for large clusters a deposition without implantation can be accomplished in the limit of very low energies, typical of 
thermal adsorption. This effect has been observed in the system ${ }^{15} \mathrm{Cu}_{147} / \mathrm{Cu}(111)$ by molecular-dynamics simulation, with no surface mixing detectable for $E_{c}=0.02 \mathrm{eV}$.

\section{CONCLUSIONS}

By using realistic interatomic potentials and moleculardynamics simulations we have investigated the behavior of Ag clusters $\left(\mathrm{Ag}_{1}, \mathrm{Ag}_{7}, \mathrm{Ag}_{19}\right)$ deposited at zero impact energy on the $\mathrm{Pd}(100)$ and $\mathrm{Pd}(111)$ substrates. We were interested (a) in establishing whether or not, and under which conditions, a cluster can land on a surface without inducing defects such as Ag substitutional impurities, Pd ejected atoms, vacancies or interstitials and (b) in assessing the role played by the adsorption energy in determining the final morphologies.

In the case of $\mathrm{Ag}_{1}$ and of a planar $\mathrm{Ag}_{7}$ keeping its structure upon landing on the surface, no site exchange or implantations are observed. On the other hand, in the case of 3D clusters implantation via a site exchange mechanism is very likely to occur, in a way correlated to the size of the cluster, the orientation of the substrate, and the exchange barrier. We proved that the energy gain associated with the deposition can be decomposed in two contributions, the first translational and the second related to the cluster shape changes. It is precisely this latter that allows us to overcome the exchange barrier favoring implantation.
To understand this phenomenon we showed that the gain of adsorption energy per atom associated with the structural transformation is larger for smaller clusters. This energy is available to promote site exchanges. However, the probability for a site exchange to occur is also related to the orientation of the substrate, which affects the height of the barrier.

We believe our findings have a general character and can be applied to metal-on-metal systems that are not expected to form surface alloys at least at low temperatures. In this context, it is worth pointing out that recently an alternative way to devise metal-on-metal deposition has been successfully implemented. ${ }^{19}$ As reported in Ref. 19, efficient dissipation of the incoming kinetic energy and substantial reduction of fragmentation has been achieved via an Argon buffer layer added on the top of a $\mathrm{Pt}(111)$ substrate prior to deposition of Ag clusters. This experiment stimulates further simulation work on cluster deposition on metallic substrate covered by noble gases, along the lines pioneered by Cheng and Landman. ${ }^{15}$

\section{ACKNOWLEDGMENTS}

We are grateful to Dr. W. Harbich and Dr. G. Vandoni for illuminating discussions. Calculations have been performed on the computers of the IDRIS computing center of CNRS located in Orsay (France).
${ }^{1}$ S. B. DiCenzo, S. D. Berry, and E. H. Hartford, Phys. Rev. B 38, 8465 (1988).

${ }^{2}$ W. Eberhardt, P. Fayet, D. M. Cox, Z. Fu, A. Kaldor, R. Sherwood, and D. Sondericker, Phys. Rev. Lett. 64, 780 (1990).

${ }^{3}$ H.-V. Roy, P. Fayet, F. Patthey, W. D. Schneider, B. Delley, and C. Massobrio, Phys. Rev. B 49, 5611 (1994).

${ }^{4}$ G. Vandoni, C. Félix, R. Monot, J. Buttet, and W. Harbich, Chem. Phys. Lett. 229, 51 (1994).

${ }^{5}$ G. Vandoni, Ph.D. dissertation, Ecole Polytechnique Féderale de Lausanne, Switzerland, 1995; C. Félix, ibid.

${ }^{6}$ C. Félix, G. Vandoni, C. Massobrio, R. Monot, J. Buttet, and W. Harbich (unpublished).

${ }^{7}$ J. M. Guglielmacci and M. Gillet, J. Cryst. Growth 57, 1558 (1982).

${ }^{8}$ S. M. Foiles, M. I. Baskes, and M. S. Daw, Phys. Rev. B 33, 7983 (1986).

${ }^{9}$ G. L. Kellogg, Phys. Rev. Lett. 76, 98 (1996).

${ }^{10}$ T. J. Raeker, L. S. Perkins, and L. Yang, Phys. Rev. B 54, 5908 (1996).
${ }^{11}$ P. Stoltze and J. K. Norskøv, Phys. Rev. B 48, 5607 (1993).

${ }^{12}$ G. Vandoni, C. Félix, and C. Massobrio, Phys. Rev. B 54, 1553 (1996).

${ }^{13}$ V. Bonačič-Koutecký, L. Čšspiva, P. Fantucci, and J. Koutecký, J. Chem. Phys. 98, 7981 (1993).

${ }^{14}$ W. D. Luedtke and U. Landman, Phys. Rev. B 44, 5970 (1991).

${ }^{15}$ H.-P. Cheng and U. Landman, J. Phys. Chem. 98, 3527 (1994).

${ }^{16}$ S. Nosé, J. Chem. Phys. 81, 511 (1984).

${ }^{17}$ We recall that in systems where a noticeable amount of transient exchanges are observed [ $\mathrm{Rh} / \mathrm{Ag}(100), \mathrm{Rh} / \mathrm{Cu}(100)$, see Ref. 10] the activation barriers $E_{\text {exc, (100) }}$ are as low as $0.26 \mathrm{eV}$, allowing the occurrence of one exchange within 1 ps at $T \sim 500 \mathrm{~K}$. This value is comparable to the local temperatures developing in the local region around the impact during the same time interval.

${ }^{18}$ C. Massobrio and B. Nacer (unpublished).

${ }^{19}$ K. Bromann, C. Félix, H. Brune, W. Harbich, R. Monot, J. Buttet, and K. Kern, Science 274, 956 (1996). 